\title{
Correção cirúrgica do aneurisma roto do seio de Valsalva: relato de dois casos
}

\author{
Lourival BONATELLI FILHO*, Amandio RAMPINELLI*, Jauro COLLAÇO*
}

RBCCV 44205-477

\begin{abstract}
Bonatelli Filho L, Rampinelli A, Collaço J - Correção cirúrgica do aneurisma roto do seio de Valsalva: relato de dois casos. Rev Bras Cir Cardiovasc 1999; 14 (4): 344-7.

RESUMO: O objetivo deste estudo é relatar a técnica cirúrgica empregada na correção de aneurisma do seio de Valsalva roto para dentro do ventrículo direito, em 2 pacientes adultos, tendo um deles, concomitantemente, leve coarctação da aorta. No primeiro caso, mulher de 22 anos com dispnéia progressiva, cuja avaliação ecocardiográfica revelou rotura do aneurisma do seio de Valsalva para dentro do ventrículo direito e coarctação da aorta leve, com gradiente de $25 \mathrm{mmHg}$. O cateterismo cardíaco confirmou o diagnóstico. No segundo caso, homem de 35 anos, com piora de dispnéia há 2 meses. Ecocardiograma e cateterismo cardíaco confirmaram o diagnóstico de aneurisma roto do seio de Valsalva para o ventrículo direito. Ambos foram operados através de esternotomia mediana; circulação extracorpórea convencional e cardioplegia sangüínea fria. No primeiro caso a valva aórtica era bivalvulada, com o aneurisma do seio de Valsalva anterior, relacionado à coronária direita, roto para dentro do VD, medindo $6 \mathrm{~mm}$ de diâmetro, foi fechado com sutura direta com fio 5-0. No segundo caso a valva aórtica era trivalvulada e o aneurisma do seio de Valsalva direito que rompeu, também, dentro do VD, media $12 \mathrm{~mm}$ de diâmetro e o fechamento foi feito com retalho de pericárdio bovino, suturado com pontos separados de polipropileno 5-0. Ambos os pacientes tiveram evolução pós-operatória sem intercorrências. Alta hospitalar ao final de uma semana. No controle ambulatorial, $120 \mathrm{e}$ 60 dias após, respectivamente, estavam assintomáticos.
\end{abstract}

DESCRITORES: Seio de Valsalva, cirurgia. Rotura aórtica, cirurgia. Aneurisma aórtico, cirurgia. Procedimentos cirúrgicos cardíacos, métodos.

\section{INTRODUÇÃO}

O aneurisma roto do seio de Valsalva é lesão cardíaca rara. A incidência é mais rara ainda nos países ocidentais do que nos orientais (1,2). Possíveis causas incluem as endocardites infecciosas, sífilis, necrose cística, aterosclerose, trauma e doença reumática ${ }^{(1-3)}$, porém a maioria dos aneurismas tem origem congênita $(1,4,5)$. A coexistência de comunicação interventricular (CIV) em significante porcentagem de pacientes reforça a natureza congênita da lesão (6). Estas lesões têm diferentes localizações e vários tipos de fístulas. Geralmente, o prognóstico é grave a menos que seja realizado o tratamento cirúrgico ${ }^{(1-3)}$.
Apresentamos os casos de 2 pacientes operados recentemente em nossa Instituição, nos quais abordamos os defeitos através de aortotomia, utilizando, entretanto, técnicas operatórias diferentes para o seu fechamento.

\section{Caso de $n^{0} 1$}

Paciente do sexo feminino, idade 22 anos, que se queixava de dispnéia progressiva iniciada há 6 meses. No decorrer do tempo, passou a sofrer dor torácica de leve para moderada intensidade, que se irradiava para o membro superior esquerdo. Negava episódios de síncopes. Ao exame físico apresenta-

Trabalho realizado no Instituto de Cardiologia da Secretaria Estadual da Saúde de Santa Catarina. Florianópolis, SC, Brasil. Apresentado ao 26ํㅡㄹ Congresso Nacional de Cirurgia Cardíaca. Fortaleza, CE, 8 a 10 de abril, 1999.

* Do Serviço de Cirurgia do Instituto de Cardiologia de Santa Catarina.

Endereço para correspondência: Lourival Bonatelli Filho. Av. Itamarati, 815. Itacorubi. Florianópolis, SC, Brasil. CEP: 88034-400. Tel: (048) 334-2029. e-mail: bonatelli@portadig.com.br 
va $\mathrm{RCR}, \mathrm{FC}=80$, PA $120 / 80$, sopro contínuo maquinário ao longo da borda esternal esquerda. Os pulsos eram simétricos. O ECG mostrava ritmo sinusal, distúrbio de condução do ramo direito. Radiografia de tórax com área cardíaca normal, sem congestão pulmonar. Ecocardiograma transtorácico mostrava câmaras, dimensões cardíacas e contratilidade normais, fluxo turbulento entre o VD e a via de saída do VE de difícil definição entre CIV e fístula Aorta-VD, mais coarctação aórtica leve, gradiente de $25 \mathrm{mmHg}$. O cateterismo cardíaco mostrou hipertensão moderada em câmaras direitas (VD = 49/ 06, $\mathrm{TP}=48 / 22$ e $\mathrm{CP}=18$ $\mathrm{mmHg}$ ), coarctação leve da aorta após a artéria subclávia esquerda e rotura de aneurisma do seio de Valsalva dentro do VD. Aorta ascendente era aumentada $++/ 4$ e o VD aumentado $+/ 4$. Instalamos a circulação extracorpórea convencional, hipotermia sistêmica moderada e cardioplegia sangüínea fria. Aortotomia. A valva aórtica era bivalvulada sem estenose ou insuficiência. No seio de Valsalva relacionado à coronária direita havia orifício de $6 \mathrm{~mm}$ de diâmetro comunicando-se com o VD. O fechamento foi por sutura direta com 2 pontos de polipropileno 5-0 com almofadas de Teflon. A operação transcorreu normalmente, assim como o período pós-operatório. A paciente recebeu alta 8 dias após a operação e encontravase em ótimas condições clínicas. Na avaliação ambulatorial a paciente estava assintomática e com ecocardiograma normal.

\section{Caso de $n^{\circ} 2$}

Homem de 35 anos, com relato de dispnéia progressiva há 6 meses e piora acentuada nos últimos 2 meses, quando procurou atendimento médico em outro hospital por apresentar, além da dispnéia, tosse produtiva e dor torácica, sem palpitações e sem antecedentes mórbidos. Tratado com antibióticos obteve melhora rápida da tosse e da dor torácica, porém, persistiu com dispnéia. Foi, então, internado em nossa Instituição para avaliação e tratamento. RCR 3 tempos, $P A=110 / 70, F C=96$, frêmito palpável na borda esternal esquerda, sopro sistodiastólico ++/4 em foco aórtico e borda esquerda do esterno, P2 hiperfonética. ECG com ritmo sinusal, sem sinais de isquemia ou sobrecarga ventricular. Exames laboratoriais normais. Ecocardiograma diagnosticou aneurisma do seio de Valsalva roto para dentro do ventrículo direito, com hiperfluxo importante na artéria pulmonar e discreto aumento do átrio esquerdo. O cateterismo cardíaco revelou

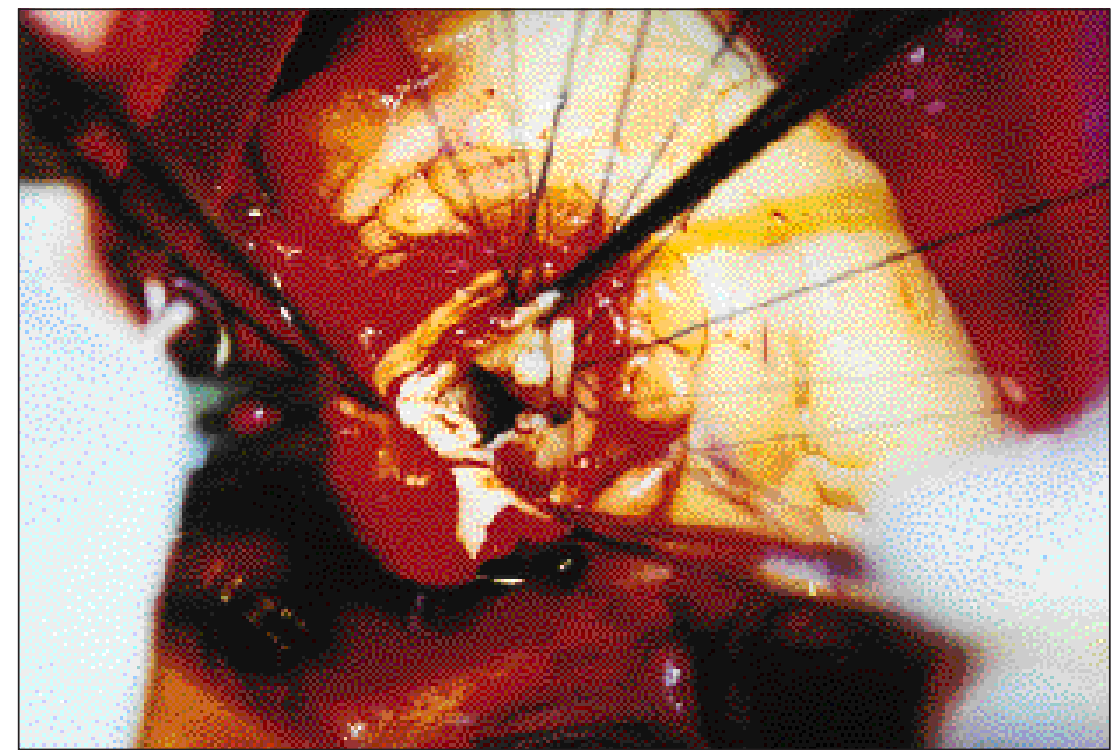

Fig. 1 - Valva aórtica trivalvulada aberta. Orifício do aneurisma coberto pela válvula coronariana direita.

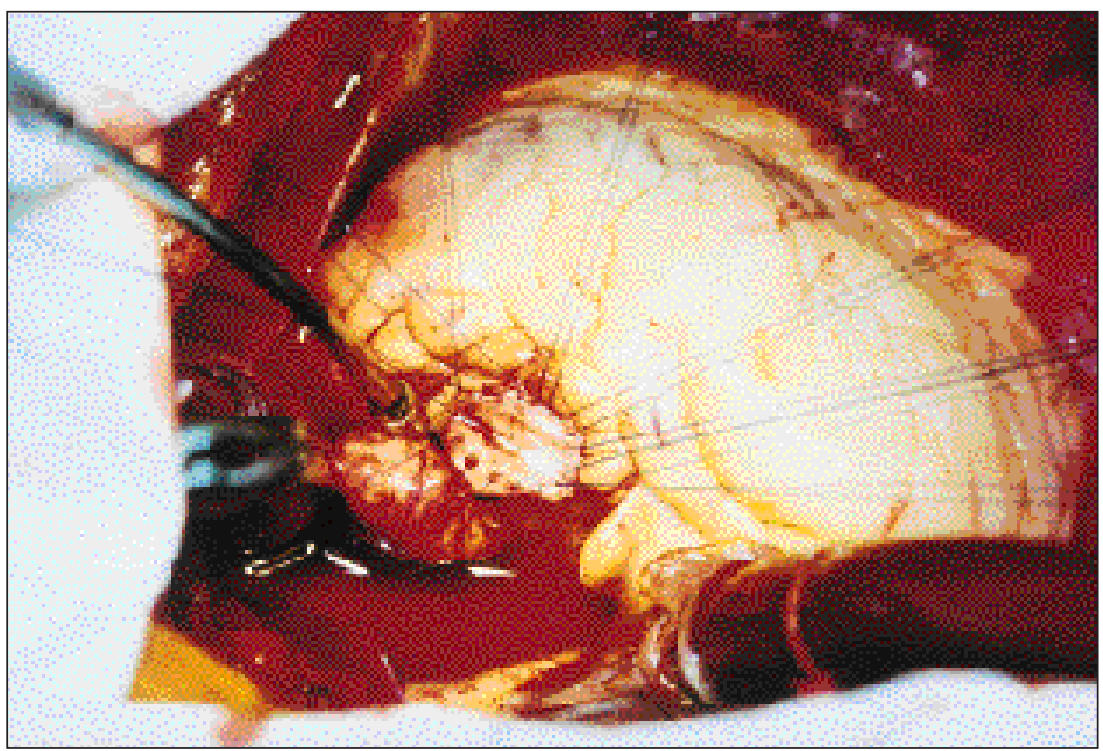

Fig. 2 - Detalhe do retalho de pericárdio bovino. 
coronárias angiograficamente sem estenoses, VE com hipocinesia difusa e moderada, VE $=105 / 35$ Aorta $=107 / 57, \mathrm{VD}=62 / 20, \mathrm{AD}=16$ e $\mathrm{CP}=20$ $\mathrm{mmHg}$. Presença de aneurisma do seio coronariano direito e fístula para o VD.

Foi submetido a tratamento cirúrgico através de esternotomia mediana, CEC com hipotermia moderada e cardioplegia sangüínea fria. Após aortotomia observamos aneurisma roto do seio de Valsalva direito no VD, sem CIV, medindo $12 \mathrm{~mm}$ de diâmetro, valva aórtica trivalvulada com válvulas espessadas nas bordas livres (Figura 1). Como a sutura direta causaria distorção das válvulas e provavelmente insuficiência aórtica, optamos pelo fechamento com retalho de pericárdio bovino suturado com pontos separados de polipropileno 5-0 (Figura 2). A operação foi concluída sem anormalidades. No pós-operatório o paciente apresentou hipertensão arterial, tratada com nitroprussiato de sódio. Alta para a enfermaria no segundo dia e alta hospitalar seis dias após a operação. No controle ambulatorial, passados dois meses, o paciente estava assintomático.

\section{COMENTÁRIOS}

A literatura tem mostrado que o aneurisma roto do seio de Valsalva é condição rara, variando entre $0,26 \%$ e $3,5 \%$ de todos os tipos de operações realizadas para tratamento das cardiopatias congênitas (1-3). A incidência tem sido maior nos países orientais do que nos ocidentais, com base nos números do Japão e Índia $(1,2,6-8)$. É mais freqüente nos homens do que nas mulheres e a grande maioria dos pacientes é operada na idade adulta $(3,6,9)$.

A etiologia mais freqüente é congênita $(4,6,9)$, cuja causa está atribuída à fraqueza na junção da média da aorta e do ânulo aórtico (5). Já MAYER et al. (2) afirmam que a endocardite infecciosa é a segunda maior causa de aneurisma roto do seio de Valsalva. Na nossa casuística, de etiologia provavelmente congênita, uma paciente tinha coarctação de aorta discreta. No entanto, esta não é uma associação freqüente. Pelo contrário, as lesões coexistentes mais freqüentemente são a CIV e a insuficiência aórtica, principalmente nos pacientes orientais. A CIV geralmente está relacionada aos aneurismas rotos do seio de Valsalva direito que rompem no VD e costuma ser do tipo supracristal ${ }^{(1,3)}$. ABE \& KOMATZU (1) notaram CIV deste tipo em $75 \%$ dos pacientes, enquanto HAMID et al. (8) encontraram $84 \%$ de CIV em seus casos, sendo $100 \%$ do tipo subarterial. Nos pacientes ocidentais a incidência de CIV e insuficiência aórtica é menor, não chegando a $50 \%(2,4)$.
Muito embora a etiologia seja geralmente congênita, é na vida adulta que os pacientes manifestam os sintomas, quase sempre expressos por dispnéia aos esforços, dor torácica e palpitações ${ }^{(6)}$. Alguns apresentam síncopes. A grande maioria dos pacientes é sintomática, a despeito do início insidioso dos sintomas $(2,9)$. Em apenas $18 \%$ dos pacientes o início dos sintomas é súbito ${ }^{(3)}$.

É comum a ausculta do sopro contínuo ao longo da borda esternal esquerda. O diagnóstico é feito através de exames ecocardiográficos e cateterismo cardíaco. Eventualmente, para melhor delineação do trajeto fistuloso, pode ser necessário o ecocardiograma intracardíaco ${ }^{(10)}$. São quatro os tipos de abordagem cirúrgica. Alguns autores $(1,9)$ abordam a câmara cardíaca na qual ocorreu a rotura do aneurisma. Outros realizam apenas a aortotomia (8) por permitir boa exposição ao reparo da fístula e manipulação da valva aórtica se esta for insuficiente. ISOMURA et al. (7), recentemente, em casos isolados, têm utilizado apenas a arteriotomia pulmonar, nos casos com valva aórtica normal e com aneurisma roto no ventrículo direito. $\mathrm{Na}$ atualidade, a abordagem preferida é a aortocameral, evitandose a ventriculotomia direita $(1,3,4,6,9)$. A vantagem reside na possibilidade de fazer a infusão direta da cardioplegia nos óstios coronários e a adequada avaliação da valva aórtica, além de diminuir os riscos de danos às válvulas da valva aórtica e distorções do anel aórtico. Atriotomia direita costuma ser a incisão complementar, uma vez que o aneurisma roto do seio de Valsalva direito, o mais freqüente, geralmente drena no VD, enquanto o aneurisma roto do seio de Valsalva não coronariano drena no átrio direito $(1,3,4,6)$. Embora muitos utilizem a sutura direta após a ressecção do saco aneurismático, que pode não existir, a recomendação de PANNU et al. (3) e VERGHESE et al. (6) é o emprego de retalho para o fechamento do aneurisma porque evita a tensão no tecido remanescente do seio de Valsalva e a distorção das válvulas da valva aórtica, diminuindo, portanto, a incidência de insuficiência aórtica tardia e a recorrência da fístula. MAYER et al. (2), LUKACS et al. (4) e van SON et al. (9) não observaram óbito hospitalar. Outros relatam, também, mortalidade baixa mesmo a longo prazo $(2,6,7,11)$. Os óbitos tardios, assim como as reoperações, habitualmente estão relacionados à troca ou plastia da valva aórtica ${ }^{(9)}$. Os resultados cirúrgicos são gratificantes e segundo $A B E$ \& KOMATZU (1) $85 \%$ dos pacientes estão em classe funcional I no seguimento médio de 11,1 anos.

A realização do diagnóstico precoce e a imediata intervenção cirúrgica com os diversos recursos técnicos atuais são os melhores caminhos para atingir maior taxa de sobrevivência e melhor qualidade de vida para os pacientes. 
Bonatelli Filho L, Rampinelli A, Collaço J - Surgical repair of ruptured sinus of Valsalva aneurysms: report of two cases. Rev Bras Cir Cardiovasc 1999; 14 (4): 344-7.

ABSTRACT: The purpose of this article is to report the surgical treatment of ruptured sinus of Valsalva aneurysms in two patients. First case was a young woman whose aneurysm originated in the right sinus and entered the right ventricle, besides a mild coarctation of the aorta. Surgical repair was carried out using cardiopulmonary bypass with moderate hypothermia. After aortotomy the ruptured sinus of Valsalva was directly closed with interrupted pledgeted sutures. The second case, a 35 years old man with symptoms for six months, including dyspnea and chest pain. Diagnosis was established by echocardiogram and cardiac catheterization findings. It was a ruptured aneurysm of the right sinus of Valsalva which drained into the right ventricle. There was no ventricular septal defect or aortic regurgitation. Surgical approach was made through ascending aortotomy. Aortic valve had three practically normal leaflets. A patch of bovine pericardium was used to close the defect by means of interrupted fine monofilament sutures. Postoperative period in both cases was uneventful. Both patients were discharged one week later. After a short follow-up, they are completely asymptomatic.

DESCRIPTORS: Sinus of Valsalva, surgery. Aortic rupture, surgery. Aortic aneurysm, surgery. Cardiac surgery procedures, methods.

\section{REFERÊNCIAS BIBLIOGRÁFICAS}

1 Abe $T$ \& Komatzu S - Surgical repair and long-term results in ruptured sinus of Valsalva aneurysm. Ann Thorac Surg 1988; 46: 520-5.

2 Mayer E D, Ruffmann K, Saggau W et al. - Ruptured aneurysms of the sinus of Valsalva. Ann Thorac Surg 1986; 42: 81-5.

3 Pannu H S, Shivaprakash K, Bazaz S et al. Geographical variations in the presentation of ruptured aneurysms of sinuses of Valsalva: evaluation of surgical repair. J Card Surg 1995; 10: 316-24.

4 Lukacs L, Bartek I, Haan A, Hankoczy J, Arvay A Ruptured aneurysms of the sinus of Valsalva. Eur $J$ Cardiothorac Surg 1992; 6: 15-7.

5 Edwards J E \& Burchell H B - The pathological anatomy of deficiencies between aortic root and the heart including aortic sinus aneurysm. Thorax 1957: 12: 125-39.

6 Verghese M, Jairaj P S, Babuthaman C, Sukumar I P,
John S - Surgical treatment of ruptured aneurysms of the sinus of Valsalva. Ann Thorac Surg 1986; 41: 284-6.

7 Isomura T, Hisatomi K, Hirano A, Satho T, Kosuga K, Ohishi K - Ruptured aneurysms of the sinus of Valsalva. J Cardiovasc Surg 1994; 35: 135-8.

8 Hamid I A, Jothi M, Rajan S, Monro J L, Cherian K M Transaortic repair of ruptured aneurysm of sinus of Valsalva. J Thorac Cardiovasc Surg 1994; 107: 1464-8.

9 Van Son J A, Danielson G K, Schaff H V, Orszulak T A, Edwards W D, Seward J B - Long-term outcome of surgical repair of ruptured sinus of Valsalva aneurysm. Circulation 1994; 90: (5 Pt 2): II 20-9.

10 Samaha F F, Lang R, Abbo K M, Carroll J D, Weinert L, Follman D F - Intracardiac ultrasonographic imaging to diagnose a ruptured sinus of Valsalva aneurysm. Am Heart J 1994; 128: 409-12.

11 Holman W L - Sinus of Valsalva aneurysms and application of surgical science to their repair. Am Thorac Surg 1993; 55: 545-50. 